\title{
Le "lingue per elaborazione" base italiana o italo-romanza in un modello socio-linguistico
} (LE) a

Con il termine "lingue per elaborazione" traduco il termine tedesco Ausbausprachen e sottintendo idiomi che si trovano nelle varie fasi di "elaborazione" (codificazione, standardizzazione), da quella più bassa (LE incipienti, come per es. il corso degli ultimi decenni) fino a quella più alta (a cui appartengono LE pienamente sviluppate, come le cinque grandi lingue romanze: il francese, l'italiano, il portoghese, il rumeno e lo spagnolo). ${ }^{1}$ Le varie traduzioni possibili del termine tedesco, contenenti il participio passato (per es. langues élaborées, langues codifiées ${ }^{2}$ e simili) non sono del tutto adeguate perché si riferiscono a lingue che hanno raggiunto il massimo grado di elaborazione. Per questa ragione avevo in un primo tempo proposto, per la terminologia italiana rispettiva, il sintagma un po' goffo "lingue elaborate o in via di elaborazione. ${ }^{\prime 3}$ Ora, essendo venuto a conoscenza di uno scritto bilingue di $\mathrm{H}$. Kloss e Grant $\mathrm{D}$. McConnell, ${ }^{4}$ considero la traduzione esatta di "langue par élaboration" molto migliore. Forse non piacerà a tutti i lettori (sit venia verbo!). Per tale ragione e anche per ragioni di brevità userò la sigla LE.

Com'è risaputo, non ogni Ausbausprache (LE) deve essere nel contempo anche una Abstandsprache, ${ }^{5}$ una "langue per distanciation" ovvero una "lingua per distanziazione" (LD). ${ }^{6}$ Le cinque grandi lingue romanze menzionate (e così pure molte altre lingue appartenenti ad altre famiglie) sono nel contempo LE e LD, ossia "elaborate" e "distanti" da tutte le altre lingue in misura sufficiente. Sono delle entità di grado autonomo sia in chiave sociolinguistica sia in chiave linguistica pura ("sistemo-linguistica"). Le possiamo notare con la sigla LED che riunisce le sigle LE e LD. ${ }^{7}$

Soltanto una LD era invece il defunto dalmatico e, fino a pochi decenni fa, il sardo. ${ }^{8}$

Soltanto LE (di grado incipiente) sarebbero il corso, il piemontese illustre e simili "giovani" LE. ${ }^{9}$ 
Non esistono sistemi linguistici che non siano né LD né LE. Un dialetto "normale," ossia un dialetto senza ambizioni standardologiche, non lo è, perché non meriterebbe il titolo di dialetto se non fosse distinto da altre unità linguistiche dello stesso livello. Da queste elucubrazioni appare chiara l'ambiguità del termine "lingua": essendo un sistema sui generis unico e irripetibile, ogni dialetto potrebbe, - se ci limitiamo ad esso - essere chiamato "lingua," nel senso più ampio del termine equivalente al contenuto del termine "sistema"; però non tutte le "lingue" sono "dialetti" ma soltanto quelle cha fanno parte di un'unità maggiore. Le LE sono per definitionem a sé stanti, ossia non fanno parte di nessuna unità maggiore; non è contraddittoria l'affermazione che il corso è una LE incipiente pur continuando a far parte del diasistema linguistico italiano, ossia pur continuando ad essere un dialetto della LD italiana.

Con questo saggio intendo gettare nuova luce sull'insieme delle LE romanze dell'epoca attuale e, nel contempo, soffermarmi su quelle LE romanze che sono di base linguistica, di "sostanza" italiana o italo-romanza.

Il modello usato sarà quello del grande sociologo e sociolinguista tedesco Heinz Kloss (1904- ) il quale lo ha applicato finora sull'insieme delle LE germaniche.

Il modello sociolinguistico klossiano è stato da me applicato, dopo una serie di ritocchi e di perfezionamenti, sulle lingue per elaborazione romanze per la prima volta in un lavoro in corso di stampa, terminato nel maggio del $1981 .^{10}$ Qui se ne dà una versione ancora più perfetta da cui risulteranno ancora meglio le caratteristiche di tutte le LE a base romanza e, in modo particolare, di quelle fra di loro che hanno una base italiana o italoromanza.

Saranno prese in considerazione, sullo sfondo panromanzo, le seguenti LE aventi sostanza italiana o italo-romanza che elenco in ordine alfabetico: il corso, il friulano, ${ }^{11}$ l'italiano, il ladino dolomitico, ${ }^{12}$ il piemontese ${ }^{13}$ e le varie LE incipienti sarde. ${ }^{14}$

Non appartengono al gruppetto menzionato le varietà regionali $^{15}$ dell'italiano standard, sebbene alcune, per es. l'italiano ticinese, ${ }^{16}$ contengano statalismi lessicali interessantissimi. Non mi occuperò neanche delle varie forme dell'italiano substandard (come italiano popolare, unitario o meno, ${ }^{17} \mathrm{l}^{\prime}$ australitaliano, ${ }^{18}$ ecc.), visto che tali idiomi, sebbene appaiano alle volte in testi narrativi, non dispongono della cosiddetta prosa letteraria o tecnica (nel largo senso della parola) ossia non si usano nella cosiddetta Sachprosa, non possiedono quelli che H. Kloss chiama sachprosalische Texte, conditio sine qua non, nel nostro secolo, di ogni LE 
anche di grado più basso. Come è risaputo, nell'epoca dei mass media, per la "normativa" e per la normalizzazione di una LE valgono più testi divulgativi, scientifici e pubblicistici delle opere liriche e narrative di più alto valore. ${ }^{19}$

Per la stessa ragione abbiamo lasciato in disparte alcuni idiomi aventi parzialmente sostanza e forma italiana, come il pidgin italiano di Eritrea ${ }^{20}$ e il cocoliche rioplatense. ${ }^{21}$ Quest'ultimo idioma molto fluido (in cui si può dire, per es. il ragazzo tiene hambre e el muchacho ha fame) viene, del resto, considerato da molti autori argentini e uruguayani piuttosto come un pidgin spagnolo. ${ }^{22}$

Se vogliamo tener conto anche di LE d'Italia romanze ma non italoromanze, si dovranno dire quattro parole sul cosiddetto arpitano, ${ }^{23}$ un dialetto francese ("franco-provenzale") valdostano che con molta euforia ostenta i propri "diritti" in tutta la zona intorno al Monte Bianco e non solo nella regione autonoma a statuto speciale Valle d'Aosta nel tentativo di "liberarsi" sia dall'italiano che dal francese letterari.

Lascerò in disparte due altre piccole LE incipienti non romanze, quali il cimbro ${ }^{24}$ e il croato molisano, ${ }^{25}$ pur essendo queste abbastanza differenti dalle "grandi" lingue loro sopraordinate quali il tedesco e il croato standard rispettivamente.

Non posso fare a meno di presentare, per coloro che non conoscono il modello di Heinz Kloss, dai suoi primordi intorno al 1930 fino alla sua fase matura e definitiva 1976-1978, il famoso reticolo composto di nove quadratini, reso pubblico per la prima volta nel $1976 .^{26}$ Tale reticolo ci permette di rappresentare graficamente l'esistenza o meno di testi non letterari (ossia della Sachprosa) di vario livello: elementare (E), liceale (L) e di ricerca (R) e su vari temi: patrii o nazionali $(\mathrm{P})$ e esteri. Quest'ultimi temi si suddividono poi in umanistici $(U)$ e temi riguardanti scienze naturali, fisiche e tecnologiche $(\mathrm{N})$.

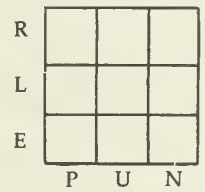

\begin{tabular}{|l|l|l|}
\hline 7 & 8 & 9 \\
\hline 4 & 5 & 6 \\
\hline 1 & 2 & 3 \\
\hline
\end{tabular}

Siccome è difficile citare i quadratini con i simboli delle loro coordinate, ho proposto la costruzione di un reticolo meno complicato con le cifre arabe, da 1 a 9, riprodotto accanto al reticolo klossiano. $^{27}$

Considero, d'accordo con $\mathrm{H}$. Kloss, come minimo necessario per l'inclusione di un idioma nell'elenco delle LE, soprattutto 
l'esistenza della prosa non-letteraria, in quantità considerevole (quanto grande debba essere questa "beträchtliche Menge" non è ancora stato accertato). Vuol dire che un idioma che dispone di testi trattanti temi patrii o nazionali sia di carattere umanistico, sia di carattere non umanistico (in tedesco: eigenbezogene Thematik), almeno al livello elementare (ossia nel primo quadratino, o nel quadratino $\mathrm{EP}$ ) è già una LE incipiente. L'esistenza di simili testi presuppone l'esistenza di un minimo di norme ortografiche e grammaticali. Invece, testi letterari (per es. quelli di F. Mistral) non si allontanano spesso dal patois del loro autore e possono essere gustati solo dagli addetti ai lavori, ossia dagli intellettuali.

Ho spiegato in altra sede le ragioni che mi hanno indotto a non far uso dei criteri praghesi, sistematizzati negli anni sessanta da P. Garvin e da M. Mathiot ${ }^{28}$ e utilizzati recentemente da G. Francescato per dimostrare che il friulano attuale è una lingua minor. ${ }^{29}$ Paola Benincà che ha recensito lo studio del Francescato si chiedeva, e con ragione, perché non vadano insigniti della stessa proprietà alcuni altri dialetti italiani con grande tradizione letteraria, con koinai piuttosto definitive ecc., senza potervi dare una giusta risposta. ${ }^{30}$ Non avendo letto le opere del Kloss non poteva ancora comprendere il ruolo dei testi non letterari (che soltanto uno dei dialetti aspiranti allo status linguistico possiede: il piemontese).

Esporrò ora il mio modello i cui presupposti sono stati spiegati in modo più esauriente e esemplificati con più particolari in altra sede. $^{31}$

Come primo criterio di questa tipologia sociolinguistica delle LE romanze userò l'opposizione che contrappone lingue soppiantatrici (con questo neologismo, sit venia verbo, denomino le lingue particolarmente espansive a danno di altre lingue sorelle, traducendo il termine klossiano, usato una sola volta, nel 1952, di Verdrängesprachen). ${ }^{32}$ Tutte le LE sono più o meno espansive ma per l'ottica impiegata questa loro caratteristica è rilevante se si effettua a danno di lingue e dialetti romanzi. Le lingue vittime (lingue soppiantate) rispondono alla prima opposizione con un "minus." Occorre dirlo che esse sono molto più numerose delle prime.

Siccome l'istrumentario usato non è binario, sono possibili anche risposte ternarie ("bipolari," ossia nel contempo "plus" e "minus": \pm ). Né particolarmente aggressive, né particolarmente vittime della storia furono il catalano, il moldavo e il portoghese. Quest'ultimo ha "soppiantato," sì, parecchie lingue extraeuropee ma non lingue o dialetti romanzi.

Il secondo criterio usato è stato assai bene studiato e esemplificato in una serie di lavori di $\mathrm{H}$. Kloss e in un mio studio in corso di stampa. ${ }^{33}$ Esso si fonda sull'atteggiamento (ingl. attitude, 
franc. attitude, ted. Einstellung) dei parlanti di fronte all'idioma da loro parlato il quale, specie se coadiuvato dall'ideologia dei sostenitori della lingua di stato, può anche differire dalla verità scientifica. Se la quasi totalità dei parlanti crede che la loro lingua è un dialetto o una forma deteriore e socialmente bassa della lingua che domina nella vita pubblica, che viene insegnata in tutte le scuole ecc., allora tale convinzione sbagliata diventa una forza motrice a cui non possono opporsi i pochi studiosi e "nazionalisti" che conoscono il vero stato delle cose. Questa opposizione oppone dunque LD apparentemente dialettalizzate a idiomi che sono "veri" dialetti di determinate "lingue egemoni."

Rispondono ternariamente al quesito Nr. 2 i cinque o sei romanci della svizzera dove abbiamo una situazione irripetibile: a ogni dialetto corrisponde praticamente una "piccola" e incipiente LE!

Se una LD durante molti secoli accetta, senza resistervi, lo status di lingua apparentemente dialettalizzata, essa finirà col diventare, non solo soggettivamente, ma anche oggettivamente, un "vero" dialetto (sarà "echtdialektisiert," per usare il termine di $\mathrm{H}$. Kloss). ${ }^{34}$ Se un tale "vero" dialetto non mostra velleità "elaborazionali" (per es. il leonese), esso non figurerà sul mio diagramma.

I due sottogruppi principali ottenuti con la domanda Nr. 2 vengono ulteriormente suddivisi con l'opposizione Nr. 3. La esemplificherò con questa coppia: il piemontese-il corso. Ambidue questi idiomi sono dal punto di vista linguistico dialetti italiani. Dal punto di vista sociolinguistico essi differiscono per un tratto importante: il Kloss considera il primo come un dialetto "con tetto" (in ted. überdachte Mundart) e il secondo come un dialetto "senza tetto" [in ted. dachlose (Außen) mundart]. ${ }^{35}$ Possiamo distinguere, dunque, dialetti "coperti" e "scoperti": I bambini piemontesi hanno la possibilità di apprendere nella scuola elementare la lingua di cui il loro dialetto fa parte, quelli corsi non lo possono fare da due secoli visto che nelle scuole dell' isola, eccettuato il breve periodo dell'occupazione, veniva insegnato solo il francese (e dopo l'estensione della Legge Deixonne vi è insegnato anche il corso). Ciascuno può considerare l'influsso dell'italiano standard sul piemontese come vuole, o come nefasto, o come utile. È incontestabile però che grazie al "tetto" italiano l'intensità dell'impatto francese sul piemontese è stata minima, soprattutto se comparata a quella dell'impatto francese sul corso.

Secondo la mia opinione che ho cercato di difendere in altra sede, anche LD apparentemente dialettalizzate possono essere ulteriormente suddivise dalla stessa opposizione, presa nel senso 
largo della parola: analoga sebbene non del tutto identica al rapporto fra il piemontese e il corso è la relazione tra il creolo ${ }^{36}$ francese della Martinica e il creolo francese delle isole state francesi nel XVII secolo e poi passate sotto il dominio inglese per diventare poi indipendenti pochi anni fa: il Maurizio e le Seicelle (Seychelles). Nel primo caso esiste la possibilità, limitata solo dalla debolezza della rete scolastica in certe zone della campagna, che tutti i bambini imparino il francese, lingua romanza e come tale vicina al loro idioma natio, nel secondo caso la maggioranza dei bambini deve imparare l'inglese.

La stessa opposizione funziona infine anche tra LD apparentemente dialettalizzate non creole. Ciò è possibile a condizione di una leggera modifica del termine "coperto" e del suo antonimo "scoperto." In vari lavori ho proposto che con questa copria di termini vengano distinti non solo, come vuole $\mathrm{H}$. Kloss, dialetti $\mathrm{i}$ cui parlanti hanno (oppure non hanno) la possibilità d'imparare nella scuola elementare la lingua "sopraordinata" del loro dialetto, ma anche le lingue creole i cui parlanti hanno la possibilità di imparare (o meno) nella scuola elementare la cosiddetta "lingua base" (Ausgangssprache, Basissprache) della loro lingua, e addirittura le lingue non creole in analoghe condizioni [per esempio, i parlanti del judezmo ${ }^{37}$ non potevano imparare lo spagnolo né nessuna lingua romanza fino a un secolo fa, sparsi com'erano in terre con lingua ufficiale araba, turca, greca o slava. Appena dopo la penetrazione spagnola nel Marocco e l'irradiazione culturale francese nel Vicino Oriente si sono enucleati due tipi di judeo español: uno "coperto" simultaneamente dall'arabo e dallo spagnolo (il cosidetto haketiya) e l'altro, "coperto" simultaneamente dal francese e da una lingua balcanica o vicino-orientale (per es. il greco, il bulgaro, il serbo-croato, il turco), detto fragnol].

Esiste un'analogia, almeno fino al 1850, fra i rapporti seguenti:

$\begin{array}{lll}\text { piemontese } & - & \text { corso } \\ \text { martiniquais } & - & \text { seychellois (o mauricien) } \\ \text { aragonese } & - & \text { judeo español }\end{array}$

Siccome i parlanti sardi e friulani si sentivano e si sentono tuttora nella maggioranza come "coperti" dall'italiano, (e quelli occitanici dal francese, ecc.), lo stesso rapporto esisteva, dal punto di vista sociolinguistico, anche fra, per es.: 
Con lo "spostamento" menzionato del judeo español le sole lingue (LD) "scoperte" sono lingue creole: fra queste un posto d'onore spetta alla più sviluppata, al papiamentu, parlata in una zona dove una lingua non romanza, il neerlandese, è stata e sarà, fino alla proclamazione dell'indipendenza, la lingua ufficiale.

Ai giorni nostri, non essendo né del tutto "coperte" né del tutto "scoperte," alcune LE rispondono in maniera bipolare, ossia con un " \pm " alla terza opposizione: l'arpitano (visto che nelle scuole valdostane i bambini imparano sia il francese che l'italiano), il ladino dolomitico (i bambini imparano tale idioma ma anche l'italiano e il tedesco) e i due giudeo-spagnoli menzionati.

Si formano così nove gruppi di LE romanze, visibili sul diagramma seguente:

\section{"LINGUE PER ELABORAZIONE" ROMANZE}

\section{Criteri:}

1. "lingue soppiantatrici" - "lingue non soppiantatrici"

2. "lingue per distanziazione apparentemente dialettalizzate" — "veri dialetti"

3. "idiomi coperti" — "idiomi scoperti"

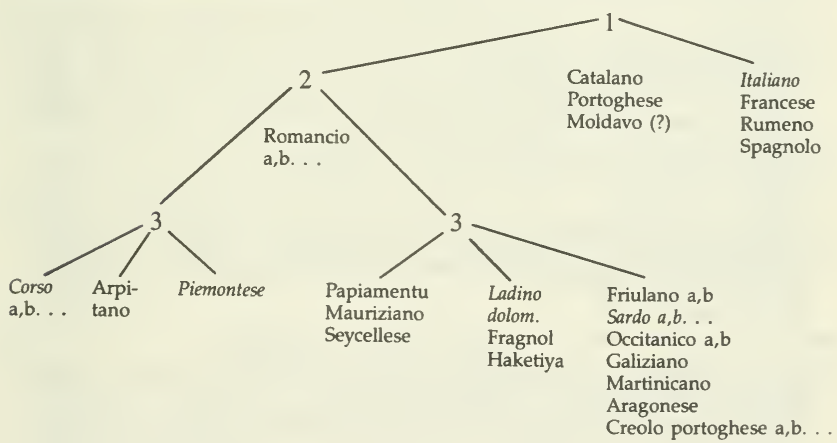

N.B. Le risposte positive si trovano sempre a destra, quelle negative a sinistra e quelle bipolari nel mezzo fra i due estremi. 


\section{NOTE}

1 Cfr. H. Kloss-G. D. McConnell, Linguistic Composition of the Nations of the World - Composition linguistique des nations du monde, vol. I (Québec, 1974), p. 32: "The term 'ausbau language' may be defined as 'language by elaboration'." Languages belonging to this category are recognized as such because of having been shaped or reshaped, molded or remolded, as the case may be, in order to become a standardized tool of literary expression. We might say that an ausbau language is called a language by virtue of its having been reshaped, i.e. by virtue of its 'reshapedness' if there were such a word. Terms such as reshaping or remolding or elaboration, by focusing on deliberate language planning, help us to avoid a misunderstanding that the term 'development' might lead to, namely that 'ausbau' might come about by that slow, almost imperceptible and quite uncontrolled growth which we are wont to call natural." Cfr. ib., p. 33 ss.

$2 \mathrm{~V}$. anche il termine langue construite, usato accanto ai precitati, da vari relatori in: W. Mackey (éd.), Minorités linguistiques et interventions. Essai de typologie. Compte rendu du Colloque sur les minorités linguistiques tenu à l'Université Laval du 15 au 18 avril 1977. Centre International de recherche sur le bilinguisme. Travaux du C.I.R.B., Études, A-15 (Québec, 1978).

3 Cfr. Ž. Muljačić, "Tipi di 'lingue in elaborazione' romanze," Incontri linguistici, VI, 2 (in corso di stampa).

4 H. Kloss-G.D. McConnell, op. cit., p. 32 ss., usano come sinonimo il semicalco langue 'ausbau'; nella parte inglese 'Ausbau' Language.

5 G. Héraud, "Notion de minorité linguistique," in W. Mackey, op. cit., p. 18, usa il termine langue-écart.

6 H. Kloss-G.D. McConnell, op. cit., p. 32 ss., usano come sinonimo il semicalco langue 'abstand'; nella parte inglese 'Abstand' Language.

7 È interessante che la componente " $E$ " si perde per prima e si ottiene per prima. Su questo "paradosso" cfr. Ž. Muljačić, "Verteidigungsstrategien gefährdeter Sprachen," [Bruxelles, 1982, (in preparazione)].

8 Non essendo informato sui più recenti sviluppi in Sardegna il Kloss, Die Entwicklung neuer germanischer Kultursprachen seit 1800 (Düsseldorf, 1978), p. 25, lo considerava come una tipica LD. Però a p. 334, esprime un'opinione più consona alla situazione attuale per cui cfr. G. Bossong, "La situation actuelle de la langue sarde. Perspectives linguistiques et politiques," Lengas, 8 (1980), 33-58.

9 Cfr. R. Petrella, La renaissance des cultures régionales en Europe (Paris, 1978); H. Kloss, Die Entwicklunk. . . cit., pp. 63,334; G. Sobiela-Caanitz, Le Piémont, in G. Héraud (éd.), Contre les États les régions d'Europe (Paris, 1973), pp. 151-62. All'ultimo momento mi è pervenuto il saggio di Kenneth Rogers, "Selected Recent Studies in Linguistic Nationalism in the Romance Languages," Canadian Review of Studies in Nationalism - Revue Canadienne des Études sur le Nationalisme, VIII, 2 (1981), 267-83.

10 Cfr. Ž. Muljačić, "Tipi di 'lingue in elaborazione' romanze."

$11 \mathrm{Cfr}$. G. Francescato, "A Sociolinguistic Survey of Friulan as a minor Language," International Journal of the Sociology of Language, 9 (Linguistic, nr. 177), 97-122; Friulan, in P. Sture Ureland, "Kulturelle und sprachliche Minderheiten in Europa. Aspekte des èropäischen Ethnolinguistik und Ethnopolitik." Akten des 4. Symposions über Sprachkontakt in Europa (Mannheim, 1980), 139-47; Ž. Muljačić "Il friulano, in base a un modello di 'Ausbaukomparatistik'," Studi in onore di Luigi Heilman (Milano, in corso di stampa).

12 Cfr. J. Kramer, Deutsch und Italienisch in Südtirol (Heidelberg, 1981), pp. 148-62; Id., recensione di F. Hoffman, Sprachen in Luxemburg (Luxemburg, 1979), Romanische Forschungen, 93, 1-2 (1981), 177-79. L'autore paragona il rifiuto del tedesco dopo il 1945 con il rifiuto dell'italiano da parte dei Ladini dopo il 1918.

13 G. Sobiela-Caanitz, Le Piémont, cit., pp. 151-62.

14 V., oltre a G. Bossong, op. cit., l'antologia commentata, edita da G. Murru Corriga, Etnia Lingua Cultura. Un dibattito aperto in Sardegna (Cagliari, 1977), 
nonché molti lavori editi o in corso di stampa della romanista viennese $R$. Rindler Schjerve.

15 Cfr. L. Canepari, Italiano standard e pronunce regionali (Padova, 1980); S.C. Sgroi, "Diaglossia, prestigio, italiano regionale e italiano standard: proposte per una nuova definizione," La ricerca dialettale, III (1981), 207-48; A.A. Sobrero-M.T. Romanello, L'italiano come si parla in Salento (Lecce, 1981); G. Tropea, Italiano di Sicilia (Palermo, 1976).

16 Cfr. O. Lurati, Dialetto e italiano regionale nella Svizzera Italiana (Lugano, 1976); id. "Lingue in contatto e stratificazione linguistica," in R. Freudestein (ed.), Language Learning. Individual needs. Interdisciplinary cooperation. Bi- and multilingualism. The Lucern Congress Report of the FIPLV (Bruxelles, 1978), pp. 199-213; H. Goebl, "Glottonymie, Glottotomie und Schizoglossie. Drei sprachpolistich bedeutsame Begriffe," Ladinia, III (1979), 7-38; id., Recensione di O. Lurati, Dialetto e italiano regionale nella Svizzera Italiana (Lugano, 1976), Vox Romanica 39 (1980), 239-41.

17 Cfr. G. Berruto, "La natura linguistica dell'italiano popolare," in G. Holtus-E. Radtke (ed.), Varietätenlinguistik des Italienischen (Tübingen, in corso di stampa); E. Radtke, "Zur Bestimmung des Italiano Popolare," Romanistisches Jahrbuch, 30 (1979), 43-58; id., "Bestimmungskriterien für das Italiano popolare," in Ch. Schwarze, (Hrsg.), Italienische Sprachwissenschaft (Tübingen), pp. 147-157; G. Sanga, "Lettere dei soldati e formazione dell'italiano popolare unitario," in M. Fontana Pieretti (ed.), Mondo popolare in Lombardia, 9. La Grande Guerra. Operai e contadini lombardi nel primo conflitto mondiale (1980), pp. 43-65; id., "Les dynamiques linguistiques de la société italienne (1961-1980): de la naissance de l'italien populaire à la diffusion des ethnicismes linguistiques," Languages, 15, No. 61 (1981), 93-115; Ż. Muljačić, "Für ein neues Modell des Architektur des Italienischen," in G. Holtus-E. Radtke (ed.), cit.

18 Cfr. F. Leoni, Vocabolario australitaliano. Con un'introduzione di Gio Andreoni (Armidale, 1981), che riporta tutti gli scritti rilevanti in materia. V. anche Muljačić, L'Italiano d'oltremare (Aspetti storici - problemi e punti nevralgici ripercussioni) (Frankfurt, 1982), in corso di stampa.

19 Cfr. H. Kloss, Die Entwicklung . . cit., pp. 28-29.

$20 \mathrm{Cfr}$. una brevissima osservazione di I. F. Hancock, "A Survey of the Pidgins and Creoles of the World," in D. Hymes (ed.), Pidginization and Creolization of Languages (Cambridge-London-New York, 1974), pp. 518-19.

21 Vedi quello che ne scrive K. Whinnon, non citato in Italia. Gli scritti del miglior conoscitore italiano del problema G. Meo Zilio-Rossi, El elemento italiano en el habla de Buenos Aires y Montevideo, tomo I (Firenze, 1970), gli sono cronologicamente anteriori.

22 Cfr. B.R. Lavandera, "Cocoliche," in Diccionario de Ciencias Sociales. UNESCO (Barcelona, 1975); id. "Sociolinguistics," in R. Posner-J. N. Green (eds.), Trends in Romance Linguistics and Philology, vol. 2 (The Hague-Paris-New York, 1981), pp. 129-228.

23 Cfr. H. Goebl, "Glottonymie. . ." cit., 7-38.

24 Cfr. Kloss, Die Entwicklung. . . cit., pp. 140-45.

$25 \mathrm{Cfr}$. Ž. Muljačić, "Su alcuni effetti del bilinguismo nella parlata dei croati molisani," Bilinguismo e diglossia in Italia (Pisa, 1973), pp. 29-37. Le altre "minoranze" nella Repubblica Italiana usano le rispettive lingue standard dei paesi d'oltre confine.

$26 \mathrm{H}$. Kloss, "Abstandsprachen und Ausbausprachen," in J. Göschel-N. O. Nail-G. Van Der Elst (Hrsg.), Zur Theorie des Dialekts (Wiesbaden, ZDL, Beihefte, N.F. 16), pp. 308-309; Id. Die Entwicklung. . . cit., pp. 37-54.

27 Ž. Muljačić, "Tipi di 'lingue in elaborazione' romanze," cit., riporta le cifre nell' ordine più solito, però non l'unico possibile, dello spiegamento: 136,248 , 57 9. Per gli esempi cfr. Haarmann, Elemente einer Soziologie der Kleinen Sprachen Europas, Band 2., Studien zur Multilingualismusforschung und Ausbaukomparatistik (Hamburg, 1979). Adesso penso sia meglio notare le cifre arabe nell'ordine qui esposto. 
28 P. Garvin-M. Mathiot, "The Urbanization of the Guarani Language: a Problem in Language and Culture," in J.A. Fishman (ed.), Readings in the Sociology of Language (The Hague-Paris, 1968), pp. 365-74.

29 G. Francescato, "A Sociolinguistic Survey of Friulan as a minor Language," cit. Vedi anche la recensione di Benincà a G. Francescato, "A Sociolinguistic Survey of Friulan as a minor Language," La Ricerca dialettale (1981), 605-08.

30 La Benincà termina la sua recensione con le seguenti obiezioni: ". . . una volta mostrato infatti che il friulano può essere considerato una lingua minore, resta da mostrare che gli altri dialetti invece non lo sono. Suppongo che, basandosi su questi parametri, il napoletano, il veneto, il lombardo, il piemontese, il ligure ecc., dialetti con grande tradizione letteraria, con forte e antica centralizzazione e situazione di macrodiglossia, con koinai piuttosto definite, ecc. ecc., siano dei candidati notevolmente interessanti per un confronto." - Il veneto degli ultimi secoli della Repubblica era un idioma usato non solo nelle opere letterarie ma anche nella storiografia, nel foro, nelle relazioni diplomatiche, negli atti, nella conversazione dotta ecc. e come tale una LE. Cfr., Th. Elwert, "L'entità ladina dolomitica e la dimensione linguistica," in L. Heilman (ed.), L'entità ladina dolomitica. Convegno interdisciplinare, Vigo di Fassa, 10-12 settembre 1976. Atti, Istituto culturale ladino (Vigo di Fassa, 1977), pp. 99-118, spec. p. 115.

31 Ž. Muljačić, "Tipi di 'lingue in elaborazione' romanze," cit.; id. "Il termine lingue distanziate apparentemente dialettalizzate (scheindialektisierte Abstansprachen) e la sua rilevanza per la sociolinguistica romanza," Mélanges Petar Skok, SRAZ, XXVI, 51-51 (Zagreb, in corso di stampa); id. "Il friulano, in base a un modello di 'Ausbaukomparatistik'," cit.; id. recensione di H. Kloss, Die Entwicklung. . . cit., Archivio glottologico italiano (Firenze, in corso di stampa).

32 H. Kloss, op. cit., pp. 161-67.

$33 \mathrm{Cfr}$. Ž. Muljačić "Il termine delle lingue distanziate apparentemente dialettalizzate," cit.

34 H. Kloss, Die Entwicklung . . . cit., pp. 67-70.

35 H. Kloss, Die Entwicklung . . . cit., pp. 60-63, V. ora anche Muljačić, "Zur Kritik des Terminus 'dachlose Aussenmundart' Beitrag zur Typologie der romanischen Ausbausprachen," Zeitschrift für Dialektologie und Linguistik (in corso di stampa).

36 H. Kloss, Die Entwicklung . . . cit., pp. 70-77, intravvede un'analogia fra i dialetti scoperti e le lingue creole tipologicamente simili ("zeitweise oder dauernd 'dachlos' gewordene kreolishe Sprachen"), senza portarla alle ultime conseguenze.

37 Per questa lingua cfr. H. Vidal Sephila, L'agonie des judéo-espagnols (Paris, 1977), passim. 